\title{
Ubiquitin-fold modifier 1 inhibits apoptosis by suppressing the endoplasmic reticulum stress response in Raw264.7 cells
}

\author{
XIAOLEI HU, QI PANG, QIONGNA SHEN, HUIFANG LIU, JIANGPING HE, \\ JING WANG, JIE XIONG, HUIJIE ZHANG and FENGLING CHEN \\ Department of Endocrinology, Shanghai 3rd People's Hospital, School of Medicine, \\ Shanghai Jiao Tong University, Shanghai 201999, P.R. China
}

Received December 12, 2013; Accepted March 21, 2014

DOI: $10.3892 / \mathrm{ijmm} .2014 .1728$

\begin{abstract}
Ubiquitin-fold modifier 1 (Ufm1) is a new member of the ubiquitin-like protein family, and its biological function remains largely unknown, particularly in macrophages. In this study, we demonstrate that Ufm1 expression is increased in diabetic mouse resident peritoneal macrophages (RPMs) and in the mouse macrophage cell line, Raw264.7, subjected to endoplasmic reticulum (ER) stress. Following treatment of the cells with the ER stress inducers, thapsigargin (TG) or tunicamycin (TM), the lentiviral short hairpin RNA (shRNA)mediated knockdown of Ufm1 increased the apoptosis of Raw264.7 cells. Furthermore, these cells had higher expression levels of immunoglobulin heavy chain-binding protein (BiP) and $\mathrm{C} / \mathrm{EBP}$ homologous protein (CHOP), which are markers of the ER stress response. The overexpression of Ufm1 induced by lentiviral infection in the Raw 264.7 cells treated with the ER stress inducers, TG or TM, resulted in the opposite effect. Taken together, our results suggest that Ufm1 is upregulated in diabetic mouse RPMs and in Raw264.7 cells in response to ER stress and that Ufm1 protects macrophages against apoptosis. Thus, Ufm1 may be a novel gene that protects against ER stress-induced apoptosis in macrophages.
\end{abstract}

\section{Introduction}

The endoplasmic reticulum (ER) is an organelle in eukaryotic cells that is involved in the synthesis, maturation, quality control and trafficking of a wide range of proteins $(1,2)$. Disruptions in ER function resulting from increased protein synthesis, misfolded protein overload, changes in calcium concentration or hypoxia induce ER stress and activate the ER stress response [also known as the unfolded protein response (UPR)] to restore

Correspondence to: Professor Fengling Chen, Department of Endocrinology, Shanghai 3rd People's Hospital, School of medicine, Shanghai Jiao Tong University, 280 Mohe Road, Shanghai 201999, P.R. China

E-mail: cf11993@126.com

Key words: ubiquitin-fold modifier 1, endoplasmic reticulum stress, apoptosis, macrophage
ER homeostasis and protect the cell. Mechanisms activated during the ER stress response include translational attenuation, activation of ER chaperones, such as immunoglobulin heavy chain-binding protein (BiP, also known as GRP78) and the degradation of unfolded proteins $(1,3)$. Chronic or unresolved ER stress, however, can lead to apoptosis. Apoptosis resulting from ER stress is mediated by factors including C/EBP homologous protein (CHOP), IRE1 $\alpha$ and caspase-12 (4-6). Accumulating evidence indicates that ER stress-induced apoptosis contributes to the pathogenesis of various diseases, such as type 2 diabetes mellitus (T2D), atherosclerosis, liver disease, neurodegenerative disorders and cancer (2,7-12).

It has also been shown that many cellular regulatory processes depend on the post-translational functions of ubiquitin and ubiquitin-like proteins (Ubls), including transcription, DNA repair, signal transduction, autophagy, cell proliferation, differentiation, apoptosis, ER regulation, inflammation, antigen processing and stress responses (13-15). Ubiquitin-fold modifier 1 (Ufm1) has recently been identified as a novel Ubl with a molecular mass of $9.1 \mathrm{kDa}$, and it appears to have a similar tertiary structure to ubiquitin, despite having little amino acid sequence identity (16). Similar to the process of protein ubiquitination, Ufm 1 is first synthesized in a pro-form and cleaved at the $\mathrm{C}$ terminus by the specific cysteine proteases, UfSP1 and UfSP2, to expose the conserved glycine residue that is essential for its subsequent conjugating reactions (17). The mature form of Ufm1 is successively activated by a specific E1-activating enzyme (Uba5), an E2-conjugating enzyme (Ufc1) and an E3-ligating enzyme (Ufl1), and then it is covalently attached to a cellular target protein, such as C20orf116 (also known as UFBP1), CDK5RAP3 and mitochondrial trifunctional protein (MTP) (18-20). Finally, Ufm1 is cleaved by UfSPs from the conjugation $(16,18)$.

Previous studies have suggested that there is an association between Ufm 1 expression and the ER stress response in mice suffering from ischemic heart injury and in the pancreatic islets of an animal model of T2D (21,22). Recently, it has been shown that Ufm1 expression is induced by ER stress and protects against ER stress-induced apoptosis in the mouse pancreatic $\beta$-cell line, INS-1E (19). Moreover, ER stress upregulates the Ufm1 system in multiple cancer cell lines, and the knockdown of the Ufm1 system results in the activation of the UPR (23). These results indicate that Ufm1 is linked to ER stress. 
Macrophages are found in all tissues and have been shown to play important roles in development, metabolic homeostasis, tissue repair and immunity (24). These findings also indicated that macrophages play important roles in T2D and atherosclerosis, and a number of studies have focused on the role of macrophage ER stress-induced apoptosis in diabetic macrovascular diseases, such as atherosclerosis (25-28). The expression pattern and potential biological function of Ufm1 in macrophages under ER stress, however, remains unclear. Therefore, in the present study, we evaluated Ufm1 expression in diabetic mouse resident peritoneal macrophages (RPMs), as well as the effects of Ufm1 on the ER stress response in the cultured mouse macrophage cell line, Raw264.7. Furthermore, the lentiviral short hairpin RNA (shRNA)-mediated knockdown of Ufm1 increased the apoptosis of Raw 264.7 cells exposed to ER stress inducers and these cells had higher expression levels of $\mathrm{BiP}$ and $\mathrm{CHOP}$, which are markers of the ER stress response. The overexpression of Ufm1 by the lentiviral infection of Raw264.7 cells treated with ER stress inducers resulted in the opposite effect. Thus, our results demonstrate that Ufm1 suppresses macrophage apoptosis by inhibiting the ER stress response in vitro.

\section{Materials and methods}

Materials. Dulbecco's modified Eagle's medium (DMEM), fetal bovine serum (FBS), penicillin, streptomycin, phosphatebuffered saline (PBS) and puromycin were purchased from Gibco BRL (Grant Island, NY, USA). Lipofectamine 2000, Opti-MEM and TRIzol reagent were obtained from Invitrogen (Carlsbad, CA, USA). The GoScript ${ }^{\mathrm{TM}}$ Reverse Transcription kit and the GoTaq ${ }^{\circledR}$ qPCR Master mix kit were purchased from Promega Corp. (Madison, WI,USA). Ethylenediaminetetraacetic acid (EDTA), trypsin, thapsigargin (TG) and tunicamycin (TM) were obtained from Sigma-Aldrich (St. Louis, MO, USA).

Animals. For our experiments, 8 - to 12 -week-old male $\mathrm{db} / \mathrm{db}$ $\left(\mathrm{C} 57 \mathrm{BL} / 6 \mathrm{~J}-\mathrm{Lepr}^{\mathrm{db}} / \mathrm{Lepr}^{\mathrm{db}}\right.$ ) mice and littermate control $\mathrm{db} / \mathrm{m}$ (C57BL/6J background) mice were purchased from the Model Animal Research Center of Nanjing University (Nanjing, China) and fed standard chow (LabDiet 5001; LabDiet, St. Louis, MO, USA). All the mice were kept in a specific pathogen-free facility and allowed free access to food and water. The experiments were performed in strict accordance with the Institutional Guidelines for the Care and Use of Laboratory Animals of Shanghai Jiao Tong University (Shanghai, China).

Isolation and culture of mouse RPMs. RPMs from $\mathrm{db} / \mathrm{db}$ mice and $\mathrm{db} / \mathrm{m}$ mice were isolated 3 days following an intraperitoneal injection of $4 \%$ thioglycollate solution, as previously described (29). Briefly, approximately $5 \mathrm{ml}$ of cold serum-free DMEM were injected intraperitoneally after the mice under were euthanized ether anesthesia. Following a gentle massage, the peritoneal fluid containing the cells was harvested and centrifuged at $700 \mathrm{x} \mathrm{g}$ for $5 \mathrm{~min}$ at $4^{\circ} \mathrm{C}$. The pellet was resuspended in $5 \mathrm{ml}$ of DMEM containing 10\% FBS, $100 \mathrm{U} / \mathrm{ml}$ penicillin and $100 \mu \mathrm{g} / \mathrm{ml}$ streptomycin and then cultured at $37^{\circ} \mathrm{C}$ in a $60-\mathrm{mm}$ dish (Corning Life Sciences, Oneonta, NY, USA) for $1 \mathrm{~h}$. Only the attached macrophages were used for the following experiments.
Cell culture. The human embryonic kidney 293T (HEK293T) cell line and mouse macrophage cell line, Raw264.7, were obtained from the American Type Culture Collection (ATCC; Rockville, MD, USA). The cells were cultured in DMEM supplemented with $10 \% \mathrm{FBS}, 100 \mathrm{U} / \mathrm{ml}$ penicillin and $100 \mu \mathrm{g} /$ $\mathrm{ml}$ streptomycin in a humidified atmosphere of $5 \% \mathrm{CO}_{2}$ at $37^{\circ} \mathrm{C}$. When the cells reached approximately 70-80\% confluency, they were dissociated with trypsinization and subcultured.

$R N A$ isolation and quantitative reverse transcription-polymerase chain reaction ( $q R T-P C R$ ). The cells were collected and washed with ice-cold PBS. Total RNA was isolated from the cells using TRIzol reagent. cDNA was synthesized using the GoScript ${ }^{\mathrm{TM}}$ Reverse Transcription System according to the manufacturer's instructions and then used as the template for qPCR. The GoTaq ${ }^{\circledR}$ qPCR Master mix kit was used for the qPCR assays. The thermal cycling conditions consisted of $2 \mathrm{~min}$ at $95^{\circ} \mathrm{C}$, followed by 40 cycles of $15 \mathrm{sec}$ at $95^{\circ} \mathrm{C}$ and 1 min at $60^{\circ} \mathrm{C}$. Each sample was run in duplicate on an ABI ViiA 7 Real-Time PCR system and analyzed by the relevant software. The $2^{-\Delta \Delta C T}$ method was used to analyze the relative changes in gene expression. Either GAPDH or $\beta$-actin was used as an internal standard. The following primers for each target gene were used: Ufm1 forward, 5'-TTCCTG CAGCTACAAGTGCG-3' and reverse, 5'-TCCAACTCGGT CTCTAGGAATGAT-3'; $\beta$-actin forward, 5'-TGTGACGTT GACATCCGTAAAGAC-3' and reverse, 5'-TCCACACAGAG TACTTGCGCTC-3'; BiP forward, 5'-GAGTTCTTCAA TGGCAAGGAGC-3' and reverse, 5'-GGACAAACATCAA GCAGTACCAGAT-3'; CHOP forward, 5'-CTCATCCCCA GGAAACGAAGAG-3' and reverse, 5'-TTGGGATGTGCG TGTGACC-3'; and GAPDH forward, 5'-TGGTGAAGGT CGGTGTGAAC-3' and reverse, 5'-GCTCCTGGAAGAT GGTGATGG-3';

Western blot analysis. The cells were harvested in RIPA lysis buffer supplemented with phenylmethanesulfonyl fluoride (PMSF) and protease inhibitor cocktail. Proteins were separated by $10-15 \%$ SDS-PAGE, transferred onto PVDF membranes, and stained with the following antibodies: anti-Ufm1 (1:10,000; Abcam, Cambridge, MA, USA), anti-BiP (1:1,000), anti-CHOP (1:1,000), anti-cleaved caspase-3 $(1: 1,000)$ (all from Cell Signaling Technology, Inc., Danvers, MA, USA), anti-GAPDH (1:5,000; Santa Cruz Biotechnology, Inc., Santa Cruz, CA, USA) and anti- $\beta$-actin (1:10,000; Cell Signaling Technology). Secondary antibodies conjugated to horseradish peroxidase and ECL western blot analysis reagents (Millipore Corp., Billerica, MA, USA) were used for detection. Immunoreactive bands were quantified using Gel-Pro 32 software (Media Cybernetics, Inc., Rockville, MD, USA).

Construction of recombinant lentivirus and cell infection. Three candidate small interfering RNA (siRNA) sequences for mouse Ufm1 were designed following the procedure from the Dharmacon siDESIGN Center: siRNA-1 (5'-CGGCTCAGAA CTGAGAATCAT-3'), siRNA-2 (5'-CGCCGTTCACAGCAGT GCTAA-3'), and siRNA-3 (5'-AGTTGGAAGCTGCTAAT ATAT-3'). Scrambled siRNA (5'-TTCTCCGAACGTGTC ACGT-3') was used as the negative control. shRNAs that corresponded to the siRNA sequences for Ufm1 and the 
A

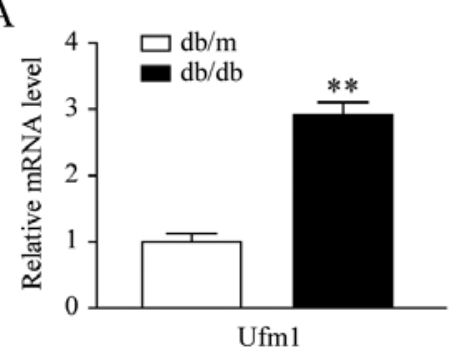

$\mathrm{D}$

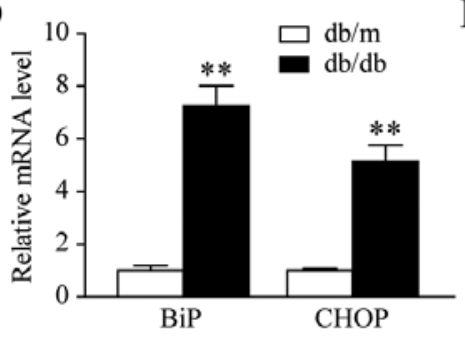

B

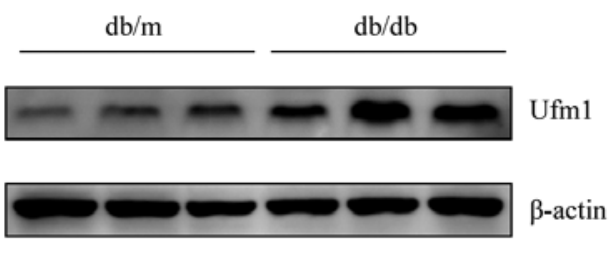

E

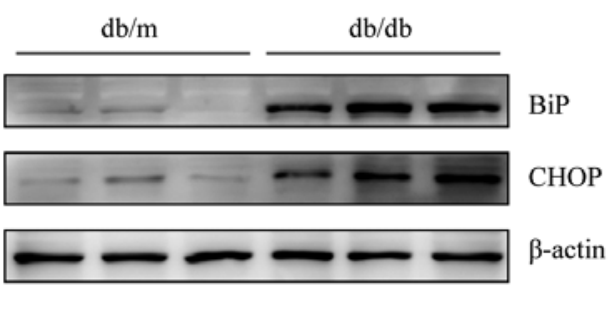

$\mathrm{C}$

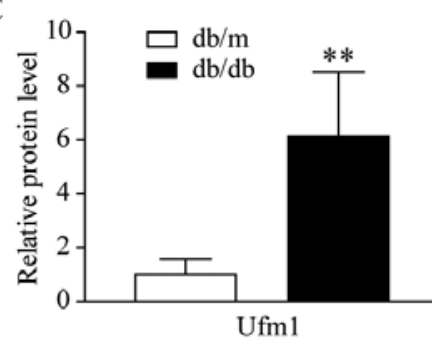

$\mathrm{F}$

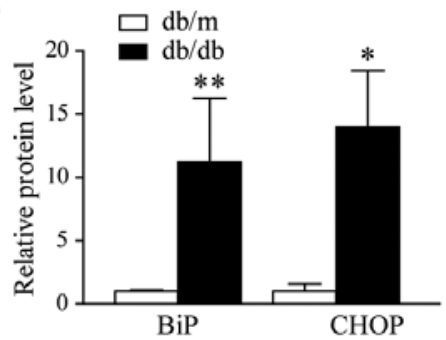

Figure 1. Increase in ubiquitin-fold modifier 1 (Ufm1) expression in the resident peritoneal macrophages (RPMs) of db/db mice. (A-C) mRNA and protein expression levels of Ufm1, (D-F) immunoglobulin heavy chain-binding protein (BiP) and C/EBP homologous protein (CHOP) in RPMs from db/m and db/db mice. $\beta$-actin was used as an internal control. Data are presented as the means \pm standard deviation $(\mathrm{SD}), \mathrm{n}=3-6 ;^{*} \mathrm{p}<0.05,{ }^{* *} \mathrm{p}<0.01 \mathrm{vs}$. db/m.

negative control were then generated. These shRNAs were inserted into the lentiviral vector, pFU-GW-RNAi (Genechem Co., Ltd., Shanghai, China). The vectors containing the Ufm1-shRNA sequences were designated as pFU-GWRNAi-Ufm1 and the vector containing the negative control was designated as pFU-GW-RNAi-NC. Lentiviruses were generated by the triple transfection of $80 \%$ confluent HEK293T cells with the modified pFU-GW-RNAi plasmid and pHelper 1.0 and pHelper 2.0 helper plasmids (Genechem Co.) using Lipofectamine 2000 according to the manufacturer's instructions. Lentiviruses were harvested $72 \mathrm{~h}$ after transfection. The lentiviruses were purified using ultracentrifugation and the titers of the lentiviruses were determined. The Raw264.7 cells were infected with either the Ufm1-shRNA lentivirus (Lv-shUfm1) or the negative control lentivirus ( $\mathrm{Lv}$-shNC).

The sequence of the mouse Ufm1 gene was obtained from GenBank (NM_026435) and oligonucleotides were designed and synthesized based on this sequence. A restriction enzyme site (NotI and NsiI) was added to each end of the oligonucleotides. PCR was performed to connect the synthesized oligonucleotides into a complete sequence. Following a NotI/NsiI (Fermentas, Glen Burnie, MD, USA) digestion, the produced fragment was ligated into the pLV5 vector (GenePharma Co.), producing the pLV5-UFM1 construct. The pLV5 vector was used as a negative control. The lentiviral vector (pLV5-UFM1 or pLV5) was added to Opti-MEM, and then packing plasmids (pGag/Pol, pRev, pVSV-G; GenePharma Co.) and Lipofectamine 2000 were added sequentially. The resulting mixture was co-transfected into the HEK293T cells, which were incubated for $72 \mathrm{~h}$. The supernatant was collected, centrifuged and filtered, and the titers of the lentiviruses were determined. The Raw264.7 cells were then infected with either the Ufm1 lentivirus (Lv-Ufm1) or the negative control lentivirus ( $\mathrm{Lv}-\mathrm{NC})$.

Detection of apoptosis by flow cytometry. Apoptosis was analyzed by double staining with Annexin V-PE and 7-AAD
(BD Biosciences, Franklin Lakes, NJ, USA), which was detected by a FACSCalibur flow cytometer (BD Biosciences). Briefly, the cells were stained with $100 \mu$ l binding buffer containing $5 \mu \mathrm{l}$ Annexin $\mathrm{V}-\mathrm{PE}$ and $5 \mu \mathrm{l}$ 7-AAD at room temperature in the dark for 10-15 min. The data were analyzed using CellQuest software (BD Biosciences).

Statistical analysis. All data are expressed as the means \pm standard deviation (SD) of 2 or 3 independent experiments. The Student's t-test was used to evaluate the differences in each group using SPSS 17.0 software. A value of $p<0.05$ was considered to indicate a statistically significant difference.

\section{Results}

Increase in Ufml expression in the RPMs of db/db mice. To investigate the functional role of Ufm1 in T2D macrophages, we determined the Ufm1 mRNA and protein expression levels in the RPMs from the $\mathrm{db} / \mathrm{db}$ mice and the littermate controls, $\mathrm{db} / \mathrm{m}$ mice. We first identified the RPMs using the previously reported surface antigen $\mathrm{F} 4 / 80^{+}$detection technique (29). Total RNA and protein were extracted from the RPMs of the $\mathrm{db} / \mathrm{db}$ and $\mathrm{db} / \mathrm{m}$ mice. The results from $\mathrm{qRT}$-PCR and western blot analysis showed that there was a significant increase in the Ufm1 mRNA and protein expression levels (Fig. 1A-C). We also determined the expression levels of $\mathrm{BiP}$ and $\mathrm{CHOP}$, which are markers of the ER stress response. As shown in Fig. 1D-F, their expression levels in the RPMs from the $\mathrm{db} / \mathrm{db}$ mice were higher than those from the $\mathrm{db} / \mathrm{m}$ mice. These results indicated that the Ufm1 mRNA and protein levels were higher in the diabetic mice and that the ER stress response was activated in the RPMs from the $\mathrm{db} / \mathrm{db}$ mice.

Ufm1 expression is upregulated by ER stress inducers in Raw264.7 cells. It has been reported that the Ufm1 expression level is higher in animal models and cultured cells under ER stress $(19,21-23)$; however, the effects of ER stress on Ufm1 

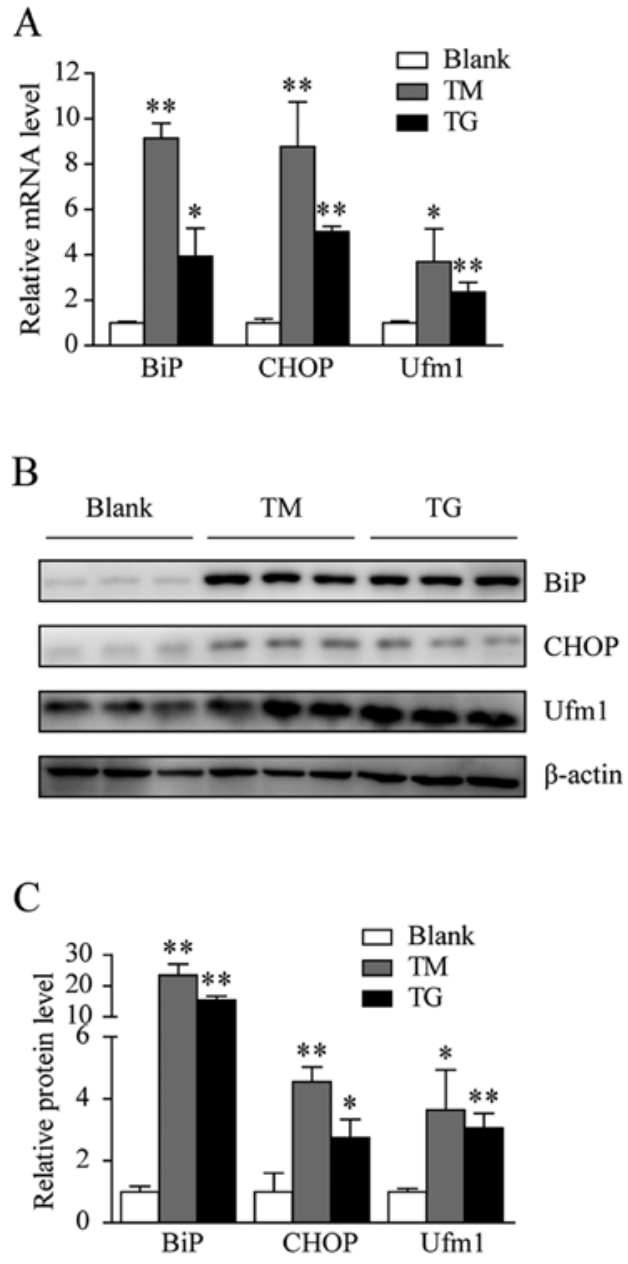

Figure 2. Ubiquitin-fold modifier 1 (Ufm1) expression is upregulated by endoplasmic reticulum (ER) stress inducers in Raw264.7 cells. Ufm1, binding immunoglobulin protein $(\mathrm{BiP})$ and $\mathrm{C} / \mathrm{EBP}$ homologous protein $(\mathrm{CHOP})$ mRNA and protein expression levels were detected by (A) qRT-PCR and (B and C) western blot analysis, after the Raw264.7 cells were treated with $0.5 \mu \mathrm{M}$ thapsigargin (TG) or $8 \mu \mathrm{g} / \mathrm{ml}$ tunicamycin (TM) for $12 \mathrm{~h}$. $\beta$-actin was used as an internal control. Data are presented as the means \pm standard deviation (SD), $\mathrm{n}=6$; ${ }^{*} \mathrm{p}<0.05,{ }^{* *} \mathrm{p}<0.01$ vs. blank. Blank, untreated cells.

expression in macrophages remain unknown. In this study, to investigate the association between Ufm1 expression and macrophage ER stress, we examined whether Ufm1 expression is affected by ER stress in Raw264.7 cells. We used TG, an inhibitor of $\mathrm{ER} \mathrm{Ca}^{2+}$ ATPase, and TM, an inhibitor of glycosylation, to induce the ER stress response in Raw264.7 cells. As shown in Fig. 2A, Ufm1 mRNA expression increased by $>2$-fold in the Raw 264.7 cells treated with $0.5 \mu \mathrm{M}$ TG for $12 \mathrm{~h}$ and by approximately 4 -fold in those treated with $8 \mu \mathrm{g} / \mathrm{ml} \mathrm{TM}$ for $12 \mathrm{~h}$. In addition, the Ufm1 protein level increased (Fig. 2B and C). To confirm that the Raw264.7 cells treated with either TG or TM were under ER stress, we also examined the BiP and CHOP expression levels. As expected, their expression levels had markedly increased (Fig. 2A-C), and this result is consistent with the results previously discussed in this study. Taken together, these data suggest that an upregulation in Ufm1 expression is associated with the ER stress response.

ER stress-induced apoptosis increases following the knockdown of Ufm1 expression in Raw264.7 cells. Apoptosis is triggered
A $\mathrm{NC}$

KD
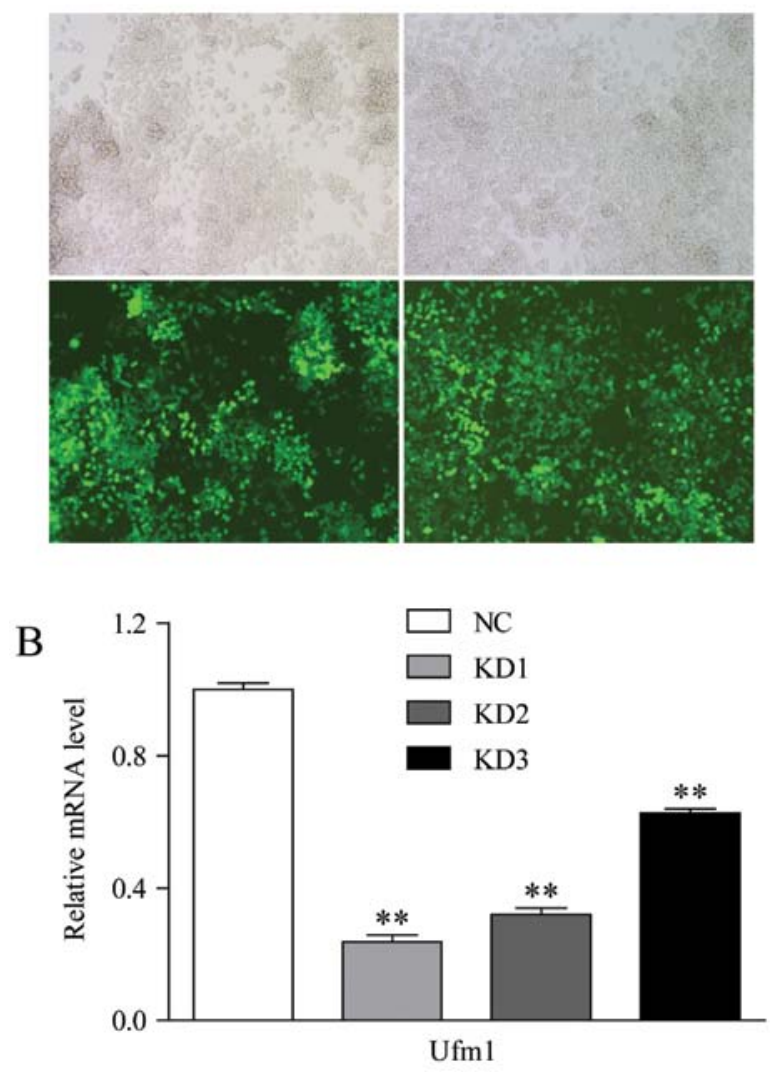

$\mathrm{C}$

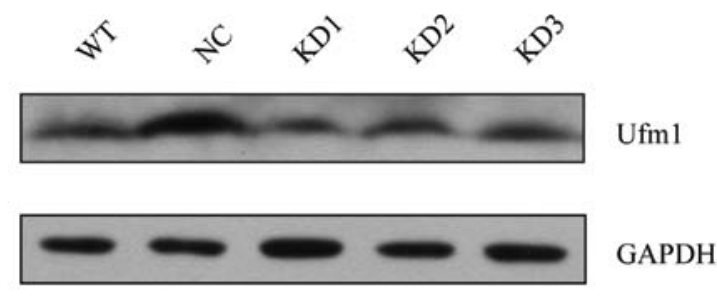

Figure 3. Verification of ubiquitin-fold modifier 1 (Ufm1) knockdown efficiency following the lentiviral infection of Raw264.7 cells. (A) Raw264.7 cells were transfected with lentivirus containing short hairpin RNA (shRNA) targeting the Ufm1 gene (Lv-shRNA-Ufm1) or negative lentivirus (Lv-shRNA-NC). After 5 days of transfection, the infection efficiency of the lentivirus was estimated to be approximately $90 \%$ as determined by the percentage of cells expressing eGFP protein by fluorescence microscopy (magnification, $\mathrm{x} 100$ ). (B and C) Knockdown efficiency of Ufm1 was analyzed by (B) qRT-PCR and (C) western blot analysis. GAPDH was used as an internal control. Data are presented as the means \pm standard deviation $(\mathrm{SD}), \mathrm{n}=3 ;{ }^{* *} \mathrm{p}<0.01$ vs. NC. WT, wild-type normal cells; $\mathrm{NC}$, negative control cells; $\mathrm{KD}$, cells in which Ufm1 was knocked down with the 3 shRNAs.

when the ER stress response fails to restore ER homeostasis. To further analyze the contributions of Ufm1 to the regulation of macrophage ER stress, as well as the effects of a reduced Ufm1 expression level on macrophage function, we examined the ER stress response and ER stress-induced apoptosis in Raw264.7 cells in which Ufm1 expression had been knocked down by lentivirus-mediated shRNA. Following viral infection, $>90 \%$ of the cells were eGFP-positive, which indicated a high efficiency of infection (Fig. 3A). Among the 3 shRNAs examined, shUfm1-1 was the most effective in reducing the Ufm1 expression level (Fig. 3B and C). Therefore, this shRNA sequence was selected for further study. According to the results from 
A

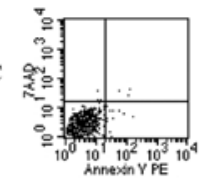

KD

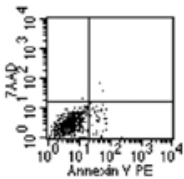

Blank
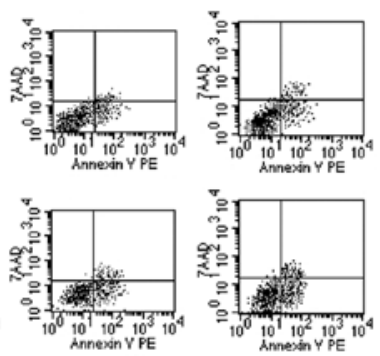

TG

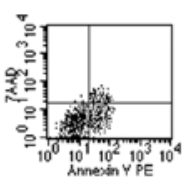

TM

C

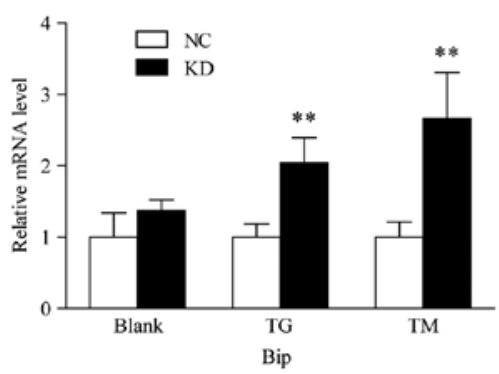

E

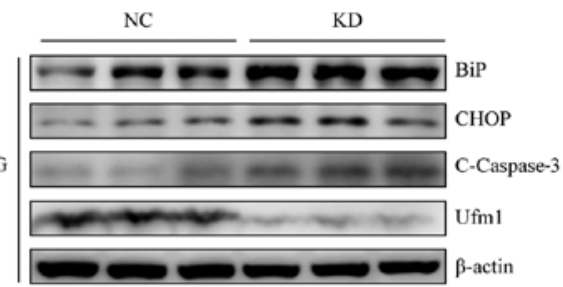

G

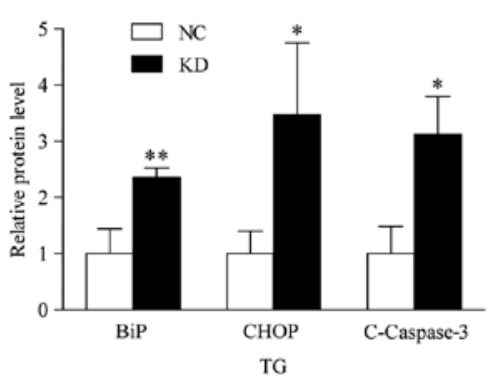

B

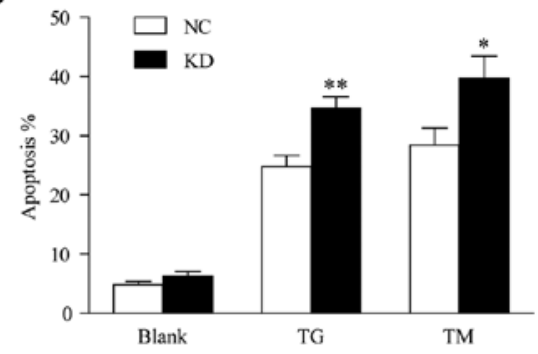

D

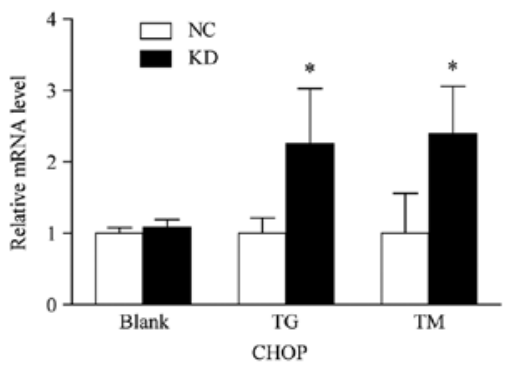

F

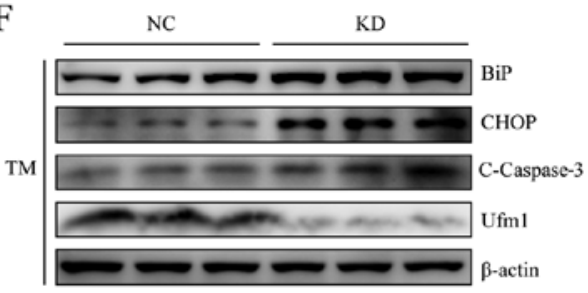

$\mathrm{H}$

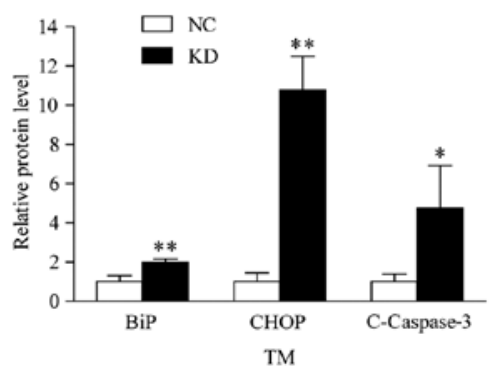

Figure 4. Endoplasmic reticulum (ER) stress-induced apoptosis increases after the knockdown of ubiquitin-fold modifier 1 (Ufm1) expression in Raw264.7 cells. The most effective shRNA to knockdown Ufm1 was selected and the cells and negative control cells were treated with $0.5 \mu \mathrm{M}$ thapsigargin (TG) or $8 \mu \mathrm{g} / \mathrm{ml}$ tunicamycin (TM) for $12 \mathrm{~h}$. (A) Representative images of flow cytometry for the analysis of apoptosis by staining with Annexin V-PE and 7-AAD. (B) The percentage of apoptotic cells was determined according to the number of cells expressing Annexin-V. (C and D) Immunoglobulin heavy chain-binding protein $(\mathrm{BiP})$ and $\mathrm{C} / \mathrm{EBP}$ homologous protein $(\mathrm{CHOP})$ mRNA expression levels were detected by qRT-PCR. (E-H) BiP, CHOP and cleaved-caspase-3 protein levels were detected by western blot analysis. $\beta$-actin was used as an internal control. Data are presented as the means \pm standard deviation (SD), $n=3-6$; ${ }^{*} \mathrm{p}<0.05,{ }^{* *} \mathrm{p}<0.01$ vs. NC. Blank, untreated cells; NC, negative control cells; KD, cells in which Ufm1 was knocked down; C-Caspase-3, cleaved caspase-3.

flow cytometry, decreasing the Ufm1 expression level markedly enhanced TG- and TM-induced apoptosis by 10 and $12 \%$, respectively, but it had no significant effect on the basal apoptosis when compared with the negative control (Fig. 4A and B). The sensitization of macrophages to ER stress-induced apoptosis was also confirmed by the level of cleaved caspase- 3 detected by western blot analysis (Fig. 4E-H). Furthermore, when the Ufm1 expression was knocked down, there was no effect on the expression levels of $\mathrm{BiP}$ and $\mathrm{CHOP}$ under no treatment conditions (Fig. 4C and D), but after the cells were treated with TG or TM, there was a higher expression level of BiP and $\mathrm{CHOP}$ compared with the negative control (Fig. 4C-H). These results indicate that Ufm1 knockdown sensitizes macrophages to ER stress. Taken together, these data demonstrate that Ufm1 is at least partially involved in regulating ER stress-induced apoptosis.
Overexpression of Ufm1 prevents ER stress-induced apoptosis in Raw264.7 cells. To further substantiate the effects of Ufm1 on ER stress-induced macrophage apoptosis, we constructed a lentiviral vector system that expresses Ufm1 cDNA and GFP and is puromycin resistant. The Raw264.7 cells were transfected with the Lv-Ufm1 or Lv-NC vectors, and after 4 days of transfection, $>80 \%$ of the cells expressed GFP (Fig. 5A), which indicated that the lentiviral infections were effective. After another 5 days of $4 \mu \mathrm{g} / \mathrm{ml}$ puromycin selection, the Ufm1 mRNA and protein expression levels in the Lv-Ufm1-infected cells were markedly increased, when compared with those in the Lv-NC-infected cells (Fig. 5B and C). To determine whether the overexpression of Ufm1 affects ER stress-induced macrophage apoptosis, the Lv-Ufm1 and Lv-NC cells were exposed to $0.5 \mu \mathrm{M}$ TG or $8 \mu \mathrm{g} /$ $\mathrm{ml}$ TM for $12 \mathrm{~h}$, and the expression levels of BiP, CHOP and cleaved caspase- 3 were determined. As predicted, in comparison 
A

$\mathrm{NC}$

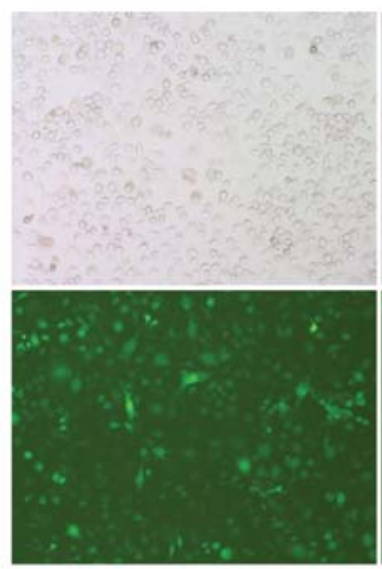

B

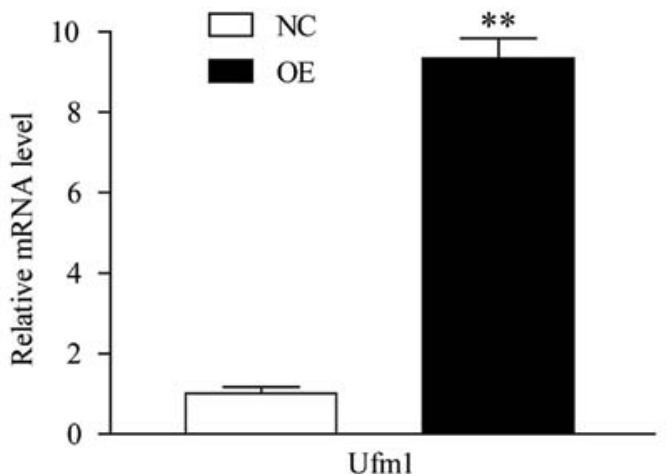

$\mathrm{C}$

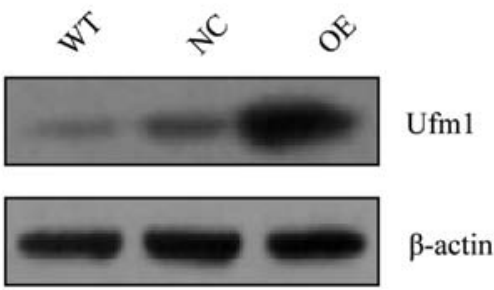

Figure 5. Verification of ubiquitin-fold modifier 1 (Ufm1) overexpression efficiency following lentiviral infection of Raw264.7 cells. Raw264.7 cells were transfected with lentivirus containing the Ufm1 gene (Lv-Ufm1) or negative lentivirus (Lv-NC). (A) After 4 days of transfection, the infection efficiency of the lentivirus was estimated to be approximately $80 \%$ as determined by the percentage of cells expressing GFP protein using fluorescence microscopy (magnification, x100). (B and C) Another 5 days of $4 \mu \mathrm{g} / \mathrm{ml}$ puromycin selection, the Ufm1 expression levels were analyzed by (B) qRT-PCR and $(\mathrm{C})$ western blot analysis. $\beta$-actin was used as an internal control. Data are presented as the means \pm stanadard deviation $(\mathrm{SD}), \mathrm{n}=3 ;{ }^{* *} \mathrm{p}<0.01 \mathrm{vs}$. NC. WT, wild-type normal cells; NC, negative control cells; OE, Ufm1 overexpressing cells.

with the negative control, the overexpression of Ufm1 attenuated macrophage ER stress and prevented the apoptosis induced by exposure to TG or TM (Fig. 6A-D).

\section{Discussion}

In the present study, we investigated the expression pattern and potential biological function of Ufm1 in macrophages. We found that Ufm1 expression was markedly upregulated in diabetic RPMs, and its expression increased in response to ER

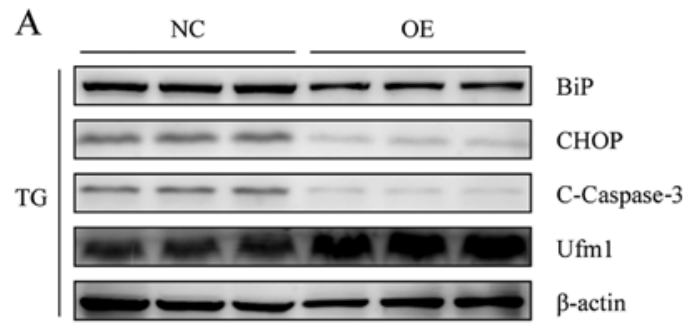

B

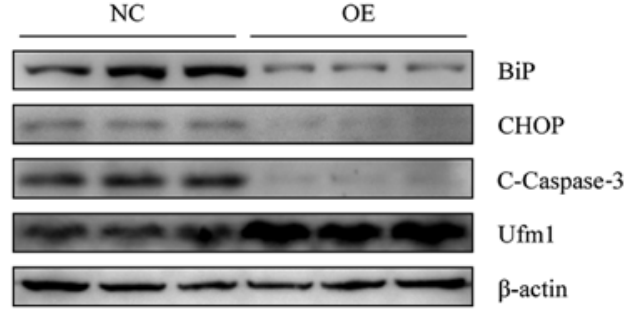

$\mathrm{C}$

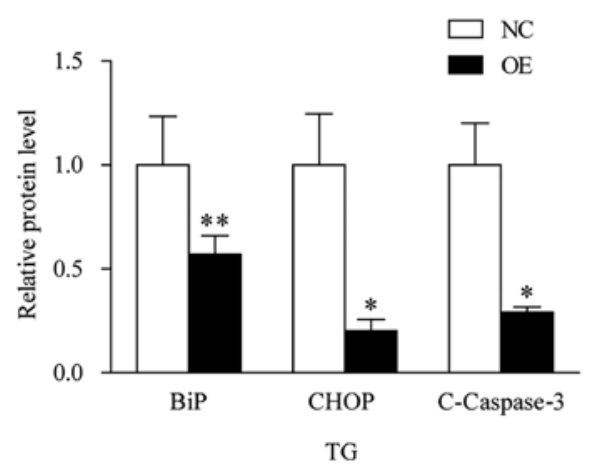

$\mathrm{D}$

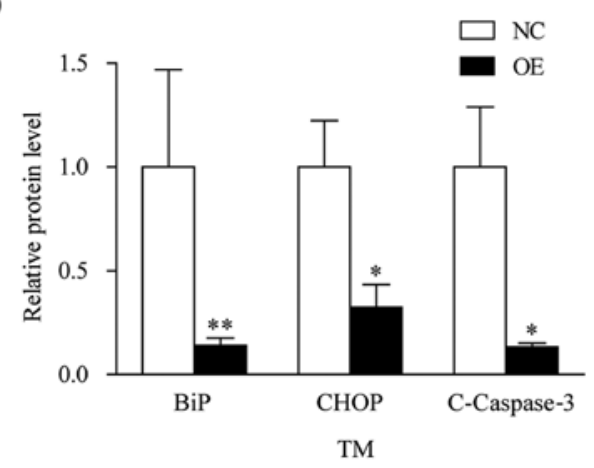

Figure 6. Overexpression of ubiquitin-fold modifier 1 (Ufm1) prevents endoplasmic reticulum (ER) stress-induced apoptosis in Raw264.7 cells (A-D) Ufm1 overexpressing and negative control cells were exposed to $0.5 \mu \mathrm{M}$ thapsigargin (TG) or $8 \mu \mathrm{g} / \mathrm{ml}$ tunicamycin (TM) for $12 \mathrm{~h}$. Immunoglobulin heavy chain-binding protein (BiP), C/EBP homologous protein (CHOP) and cleaved-caspase-3 protein levels were analyzed by western blot analysis. $\beta$-actin was used as an internal control. Data are presented as the means \pm standard deviation (SD), $n=6 ;{ }^{*} \mathrm{p}<0.05,{ }^{* *} \mathrm{p}<0.01$ vs. NC. NC, negative control cells; OE, Ufm1 overexpressing cells. C-Caspase-3, cleaved caspase-3.

stress inducers in cultured macrophages. Further investigation revealed that ER stress-induced macrophage apoptosis increased after the knockdown of Ufm1 and that the overexpression of Ufm1 attenuated ER stress-induced macrophage apoptosis.

Ufm1 is a new member of the Ubl family, whose biological functions are poorly understood, particularly in macrophages. 
It has been reported that Ufm1 is expressed in many tissues and cell lines $(16,19)$ and that an upregulation in Ufm1 expression is linked with the activation of the ER stress response in ischemic heart injury and T2D $(21,22)$. Consistent with these previous studies, our results demonstrate that Ufm1 is expressed in RPMs from normal mice and that its expression is upregulated in RPMs from diabetic mice. Additionally, the expression levels of $\mathrm{BiP}$ and $\mathrm{CHOP}$ were upregulated in the RPMs from diabetic mice compared with those from the control mice. Elevated $\mathrm{BiP}$ and $\mathrm{CHOP}$ expression levels may potentially result from insulin resistance or glucose and lipid metabolic abnormalities, which are known ER stress inducers and may contribute to impeding macrophage ER function. These findings are also consistent with those of our previous studies using cDNA microarray expression profiling in RPMs from diabetic mice (unpublished data). Chemicals such as TG and TM are commonly used to induce the ER stress response in cultured cells and animals for experimental purposes (30). In our study, we used TG and TM to induce the ER stress response in the Raw264.7 macrophage cell line. Following exposure to TG or TM, the Ufm1 mRNA and protein expression levels increased; these results are consistent with those of previous studies $(19,23)$ and the results presented above. The BiP and CHOP expression levels were also higher in the TG- or TM-treated groups, which indicated that the ER stress response was activated in these cells. Taken together, these findings suggest that Ufm1 is associated with macrophage ER stress.

It has been reported that prolonged ER stress can trigger apoptosis. CHOP is a member of the C/EBP family of bZIP transcription factors (5). Mounting evidence suggests that CHOP plays an essential role in regulating macrophage ER stress-induced apoptosis, including the release of ER calcium, the mitochondrial release of apoptogens and the activation of the death receptor, Fas (31-35). Caspase-3 is a well-known apoptosis effector, and it has been reported to be involved in ER stress-induced apoptosis (36,37). Based on our findings that Ufm1 expression was upregulated by ER stress inducers and that the Ufm1 expression pattern was similar to that of several UPR genes and the known functions of activated CHOP, we initially hypothesized that Ufm1 may be a negative regulator in macrophage ER stress-induced apoptosis and that the knockdown of Ufm1 may reduce the amount of ER stress and apoptosis observed following treatment with TG or TM. To our surprise, however, our flow cytometry and western blot analysis results revealed that the knockdown of Ufm1 expression exacerbated ER stress-induced apoptosis in the cultured macrophages. To confirm this finding, we established an Ufm1 overexpression model. The overexpressing of Ufm1 significantly reduced the BiP and CHOP expression levels, as well as the cleavedcaspase-3 expression level under ER stress conditions and reduced ER stress-induced apoptosis. These results are slightly different from those of a previous study in which there was no change in the UPR when the Ufm1 expression was knocked down in INS-1E cells, even though Ufm1 knockdown sensitized $\beta$-cells to apoptosis (19). Our results, however, are similar to the findings of a recent study by Zhang et al (23). The authors found that knocking down the expression of Ufm1 components in U2OS cells triggered the activation of the UPR and amplified the ER network (23). This discrepancy between studies may be attributed to differences in the protocols used in each study, such as the cell lines, chemical inducers, RNA interference and target gene knockdown efficiency. Additionally, Ufm1 is expressed in many tissues and cells under physiological conditions $(16,19)$, and its Uba5 plays a critical role in cell differentiation (38), which provides indirect evidence that Ufm1 may play a key role in normal cellular function and that Ufm1 knockdown may affect macrophage function. Taken together, these findings establish a link between Ufm1 and ER stress and suggest that Ufm1 is a novel protective gene whose expression is upregulated in response to ER stress-induced macrophage apoptosis. Further studies are required to elucidate the precise mechanisms involved.

In conlcusion, our study demonstrates that Ufm1 expression is involved in macrophage ER stress and that Ufm1 inhibits apoptosis by suppressing the ER stress response in macrophages. These results demonstrate the significance of Ufm1 in regulating macrophage function during ER stress, indicating that an increase in Ufm1 expression protects a compensatory response in diabetic mice and suggests that Ufm1 be considered as a potential therapeutic target against diabetes.

\section{Acknowledgements}

This study was supported by the National Natural Science Foundation of China (grant no. 81270908). The authors would like to acknowledge the expert assistance provided by Professor Jinke Cheng (Department of Biochemistry and Molecular Cell Biology, School of Medicine, Shanghai Jiao Tong University, Shanghai, China).

1

1. Schröder M and Kaufman RJ: The mammalian unfolded protein response. Annu Rev Biochem 74: 739-789, 2005.

2. Hotamisligil GS: Endoplasmic reticulum stress and the inflammatory basis of metabolic disease. Cell 140: 900-917, 2010.

3. Ron D and Walter P: Signal integration in the endoplasmic reticulum unfolded protein response. Nat Rev Mol Cell Biol 8: 519-529, 2007.

4. Nakagawa T, Zhu H, Morishima N, et al: Caspase-12 mediates endoplasmic-reticulum-specific apoptosis and cytotoxicity by amyloid-beta. Nature 403: 98-103, 2000.

5. Oyadomari S and Mori M: Roles of CHOP/GADD153 in endoplasmic reticulum stress. Cell Death Differ 11: 381-389, 2004.

6. Tabas I and Ron D: Integrating the mechanisms of apoptosis induced by endoplasmic reticulum stress. Nat Cell Biol 13: 184-190, 2011.

7. Ozcan L and Tabas I: Role of endoplasmic reticulum stress in metabolic disease and other disorders. Annu Rev Med 63: 317-328, 2012.

8. Hotamisligil GS: Endoplasmic reticulum stress and atherosclerosis. Nat Med 16: 396-399, 2010.

9. Back SH and Kaufman RJ: Endoplasmic reticulum stress and type 2 diabetes. Annu Rev Biochem 81: 767-793, 2012.

10. Kammoun HL, Chabanon H, Hainault I, et al: GRP78 expression inhibits insulin and ER stress-induced SREBP-1c activation and reduces hepatic steatosis in mice. J Clin Invest 119: 1201-1215, 2009.

11. Imai Y, Soda M and Takahashi R: Parkin suppresses unfolded protein stress-induced cell death through its E3 ubiquitin-protein ligase activity. J Biol Chem 275: 35661-35664, 2000.

12. Lee AS: GRP78 induction in cancer: therapeutic and prognostic implications. Cancer Res 67: 3496-3499, 2007.

13. Weissman AM: Themes and variations on ubiquitylation. Nat Rev Mol Cell Biol 2: 169-178, 2001.

14. Kerscher O, Felberbaum R and Hochstrasser M: Modification of proteins by ubiquitin and ubiquitin-like proteins. Annu Rev Cell Dev Biol 22: 159-180, 2006. 
15. Hochstrasser M: Origin and function of ubiquitin-like proteins. Nature 458: 422-429, 2009.

16. Komatsu M, Chiba T, Tatsumi K, et al: A novel protein-conjugating system for Ufm1, a ubiquitin-fold modifier. EMBO J 23: 1977-1986, 2004.

17. Kang SH, Kim GR, Seong M, et al: Two novel ubiquitin-fold modifier 1 (Ufm1)-specific proteases, UfSP1 and UfSP2. J Biol Chem 282: 5256-5262, 2007.

18. Tatsumi K, Sou YS, Tada N, et al: A novel type of E3 ligase for the Ufm1 conjugation system. J Biol Chem 285: 5417-5427, 2010

19. Lemaire K, Moura RF, Granvik M, et al: Ubiquitin fold modifier 1 (UFM1) and its target UFBP1 protect pancreatic beta cells from ER stress-induced apoptosis. PLoS One 6: e18517, 2011.

20. Gannavaram S, Connelly PS, Daniels MP, Duncan R, Salotra P and Nakhasi HL: Deletion of mitochondrial associated ubiquitin fold modifier protein Ufm1 in Leishmania donovani results in loss of beta-oxidation of fatty acids and blocks cell division in the amastigote stage. Mol Microbiol 86: 187-198, 2012.

21. Azfer A, Niu J, Rogers LM, Adamski FM and Kolattukudy PE: Activation of endoplasmic reticulum stress response during the development of ischemic heart disease. Am J Physiol Heart Circ Physiol 291: H1411-H1420, 2006.

22. Lu H, Yang Y, Allister EM, Wijesekara N and Wheeler MB: The identification of potential factors associated with the development of type 2 diabetes: a quantitative proteomics approach. Mol Cell Proteomics 7: 1434-1451, 2008.

23. Zhang Y, Zhang M, Wu J, Lei G and Li H: Transcriptional regulation of the Ufm1 conjugation system in response to disturbance of the endoplasmic reticulum homeostasis and inhibition of vesicle trafficking. PLoS One 7: e48587, 2012.

24. Wynn TA, Chawla A and Pollard JW: Macrophage biology in development, homeostasis and disease. Nature 496: 445-455, 2013

25. Senokuchi T, Liang CP, Seimon TA, et al: Forkhead transcription factors (FoxOs) promote apoptosis of insulin-resistant macrophages during cholesterol-induced endoplasmic reticulum stress. Diabetes 57: 2967-2976, 2008.

26. Liang CP, Han S, Li G, Tabas I and Tall AR: Impaired MEK signaling and SERCA expression promote ER stress and apoptosis in insulin-resistant macrophages and are reversed by exenatide treatment. Diabetes 61: 2609-2620, 2012.
27. Seimon TA, Nadolski MJ, Liao X, et al: Atherogenic lipids and lipoproteins trigger CD36-TLR2-dependent apoptosis in macrophages undergoing endoplasmic reticulum stress. Cell Metab 12: 467-482, 2010

28. Tabas I: The role of endoplasmic reticulum stress in the progression of atherosclerosis. Circ Res 107: 839-850, 2010.

29. Liu HF, Zhang HJ, Hu QX, et al: Altered polarization, morphology, and impaired innate immunity germane to resident peritoneal macrophages in mice with long-term type 2 diabetes. J Biomed Biotechnol 2012: 867023, 2012.

30. Yoshida H: ER stress and diseases. FEBS J 274: 630-658, 2007.

31. Ozcan L and Tabas I: Pivotal role of calcium/calmodulin-dependent protein kinase II in ER stress-induced apoptosis. Cell Cycle 9: 223-224, 2010.

32. Timmins JM, Ozcan L, Seimon TA, et al: Calcium/calmodulin-dependent protein kinase II links ER stress with Fas and mitochondrial apoptosis pathways. J Clin Invest 119: 2925-2941, 2009.

33. Li G, Mongillo M, Chin KT, et al: Role of ERO1-alpha-mediated stimulation of inositol 1,4,5-triphosphate receptor activity in endoplasmic reticulum stress-induced apoptosis. J Cell Biol 186: 783-792, 2009.

34. Thorp E, Li G, Seimon TA, Kuriakose G, Ron D and Tabas I: Reduced apoptosis and plaque necrosis in advanced atherosclerotic lesions of Apoe-/- and Ldlr-/- mice lacking CHOP. Cell Metab 9: 474-481, 2009.

35. Tsukano H, Gotoh T, Endo M, et al: The endoplasmic reticulum stress-C/EBP homologous protein pathway-mediated apoptosis in macrophages contributes to the instability of atherosclerotic plaques. Arterioscler Thromb Vasc Biol 30: 1925-1932, 2010.

36. Song L, De Sarno P and Jope RS: Central role of glycogen synthase kinase-3beta in endoplasmic reticulum stress-induced caspase-3 activation. J Biol Chem 277: 44701-44708, 2002.

37. Morishima N, Nakanishi K, Takenouchi H, Shibata T and Yasuhiko Y: An endoplasmic reticulum stress-specific caspase cascade in apoptosis. Cytochrome c-independent activation of caspase-9 by caspase-12. J Biol Chem 277: 34287-34294, 2002.

38. Tatsumi K, Yamamoto-Mukai H, Shimizu R, et al: The Ufm1-activating enzyme Uba5 is indispensable for erythroid differentiation in mice. Nat Commun 2: 181, 2011. 